\title{
Harnessing Cooperative Interactions between Thermoresponsive Aptamers and Gels To Trap and Release Nanoparticles
}

\section{Citation}

Liu, Ya, Olga Kuksenok, Ximin He, Michael Aizenberg, Joanna Aizenberg, and Anna C. Balazs. 2016. "Harnessing Cooperative Interactions Between Thermoresponsive Aptamers and Gels To Trap and Release Nanoparticles." ACS Applied Materials \& Interfaces 8 (44) (September 7): 30475-30483. doi:10.1021/acsami.6b06575.

\section{Published Version}

doi:10.1021/acsami.6b06575

\section{Permanent link}

http://nrs.harvard.edu/urn-3:HUL.InstRepos:37235080

\section{Terms of Use}

This article was downloaded from Harvard University's DASH repository, and is made available under the terms and conditions applicable to Open Access Policy Articles, as set forth at http:// nrs.harvard.edu/urn-3:HUL.InstRepos:dash.current.terms-of-use\#OAP

\section{Share Your Story}

The Harvard community has made this article openly available.

Please share how this access benefits you. Submit a story.

Accessibility 


\title{
Harnessing Cooperative Interactions Between Thermo-responsive Aptamers and Gels to Trap and Release Nanoparticles
}

Ya Liu, ${ }^{1}$ Olga Kuksenok, ${ }^{2}$ Ximin He, ${ }^{3,4}$ Michael Aizenberg, ${ }^{5}$ Joanna Aizenberg ${ }^{5,6,7}$ and Anna C. Balazs, ${ }^{1 *}$

\section{Affiliations}

1. Department of Chemical and Petroleum Engineering, University of Pittsburgh, Pittsburgh, PA, 15261, United States.

2. Materials Science and Engineering Department, Clemson University, Clemson, SC, 29634, United States.

3. Biodesign Institute, Arizona State University, Tempe, AZ, 85281, United States.

4. Materials Science and Engineering, School for Engineering of Matter, Transport and Energy, Arizona State University, Tempe, AZ, 85281, United States.

5. Wyss Institute for Biologically Inspired Engineering and Kavli Institute for Bionano Science and Technology, Harvard University, Cambridge, MA, 02138, United States.

6. Department of Chemistry and Chemical Biology, Harvard University, Cambridge, MA, 02138, United States.

7. School of Engineering and Applied Science, Harvard University, Cambridge, MA, 02138, United States.

\begin{abstract}
We use computational modeling to design a device that can controllably trap and release particles in solution in response to variations in temperature. The system exploits the thermoresponsive properties of end-grafted fibers and the underlying gel substrate. The fibers mimic the temperature-dependent behavior of biological aptamers, which form a hairpin structure at low temperatures $(T)$ and unfold at higher $T$, consequently losing their binding affinity. The gel substrate exhibits a lower critical solution temperature (LCST) and thus, expands at low temperatures and contracts at higher $T$. By developing a new dissipative particle dynamics (DPD) simulation, we examine the behavior of this hybrid system in a flowing fluid that contains buoyant nanoparticles. At low $T$, the expansion of the gel causes the hairpin-shaped fibers to extend into the path of the fluid-driven particle. Exhibiting a high binding affinity for these particles at low temperature, the fibers effectively trap and extract the particles from the surrounding solution. When the temperature is increased, the unfolding of the fiber and collapse of the supporting gel layer cause the particles to be released and transported away from the layer by the applied shear flow. Since the temperature-induced conformational changes of the fiber and polymer gel are reversible, the system can be used repeatedly to "catch and release" particles in solution. Our findings provide guidelines for creating fluidic devices that are effective at purifying contaminated solutions or trapping cells for biological assays.
\end{abstract}

Keywords: dissipative particle dynamics simulations, thermo-responsive gel, aptamers, nanoparticles, microfluidic device, catch-and-release system

\footnotetext{
*Corresponding author

Email: balazs@pitt.edu
} 


\section{Introduction}

One of the vital functions provided by microfluidic devices is the extraction of targeted particles from the fluid flowing through the system. This process is key to filtering contaminated solutions or trapping biological cells for specific assays. A fundamental challenge is creating devices that can perform these important functions relatively autonomously and repeatedly, without requiring significant human intervention to carry out the tasks. Recent studies have shown how this functionality can be achieved by harnessing biological aptamers to "catch-andrelease" biomolecules or cells in microchambers. ${ }^{1-2}$ Notably, aptamers can be driven to controllably change conformation from a folded to unfolded structure by varying the local $\mathrm{pH}$ of

the solution ${ }^{1}$ or the temperature of the system. ${ }^{2}$ The biological activity of aptamers is directly related to their structure: folded aptamers can bind and trap molecules in solution, but these bonds are broken when the aptamers unfold. Importantly, the biological activity of the aptamers can be restored by altering the $\mathrm{pH}$ or temperature to produce the folded structure. Hence, the aptamers can be used repeatedly in the catch-and-release applications. ${ }^{1-2}$

In a recently designed device, ${ }^{1}$ we matched the $\mathrm{pH}$-dependent properties of the aptamers to the dynamic behavior of a $\mathrm{pH}$-sensitive hydrogel in order to create a robust microfluidic sorting system. In this system, the aptamers were anchored to microscopic fins, which were embedded in the hydrogel, and the $\mathrm{pH}$ of the solution was dynamically altered through external pumps. By synchronizing the $\mathrm{pH}$-dependent binding strength of the aptamer with volume changes of the pH-responsive hydrogel, we could achieve the highly efficient extraction of biomolecules from the surrounding solution. Moreover, by cyclically modifying the $\mathrm{pH}$ of the solution, the system could be made to perform this function repeatedly. 
The thermo-responsive behavior of the aptamers has also been exploited to perform the selective catch-and-release of biological cells in microchambers. Here, the aptamers were bound to a solid substrate whose temperature could be carefully regulated. ${ }^{2}$ At relatively low temperatures, the aptamers folded into a hairpin-like structure and hence, could bind the cells that were driven to flow through the device. The temperature was then increased to $48^{\circ} C$, which lies above the transition temperature associated with the unfolding of the aptamers. At this elevated temperature, the aptamer-cell binding was disrupted and the cells were released to the surrounding fluid.

Herein, we use computational modeling to design a new catch-and-release system; the novelty of our system is that it exploits the synchronized, temperature-dependent behavior of both the aptamers and an underlying polymer gel. Namely, our system encompasses thermoresponsive aptamers that are directly anchored to a thermo-responsive gel, which exhibits a lower critical solution temperature (LCST). Hence, the gel swells at low temperatures and shrinks as the temperature is increased. As we show below, at low $T$, the expansion of the gel effectively pushes the anchored, hairpin-shaped chains into the path of the fluid-driven particles, and thereby enables the system to trap and extract species from solution. In this manner, the swollen gel plays an active role in optimizing the performance of the system. As $T$ is increased, the unfolding of the chain and collapse of the supporting gel enables the applied shear flow to dislodge the particles and transport them away from the layer. The shrinking of the gel at high $T$ also contributes to the utility of the system since it increases the size of the region affected by the flowing fluid.

In a device based on these principles, the gel layer would be anchored to the floor of a microfluidic chamber, with the aptamers attached to the top of this layer. A pump would drive a 
solution containing the targeted particles past this aptamer-functionalized gel. The temperature of the system could be controlled externally. ${ }^{2}$ As the system is cooled, the hairpin-shaped aptamers on the swollen gel would catch and trap the particles; as the system is heated; the unfolding of these chains on the collapsed gel would lead to the release of the trapped species. Since the temperature-induced conformational changes of the chains and polymer gel are reversible, the system can be used to repeatedly catch and release the particles.

To probe the specific mechanisms involved in trapping and releasing nanoparticles in solution via this temperature-sensitive system, we use dissipative particle dynamics (DPD) simulations, ${ }^{3}$ which can be viewed as a coarse-grained molecular dynamics (MD) approach. The other novel aspect of our study is that we integrate a DPD method for simulating the conformations of proteins ${ }^{4}$ with a recently developed DPD model for thermo-responsive gels. ${ }^{5}$ The former method ${ }^{4}$ reproduces transitions among coil-like, globular, $\alpha$-helical, and $\beta$-hairpin configurations of model peptides. The latter model ${ }^{5}$ accurately captures the temperature-induced volume phase transition for a LCST polymer gel. ${ }^{6}$ By integrating these two DPD schemes, we can probe the concerted response of the gel and aptamer to variations in temperature.

A distinctive feature of the DPD method is that it employs a soft-core repulsive potential between the beads, which represent clusters of molecules. Hence, one can use larger time steps in the integration scheme and thus, sample larger length and time scales than are possible via MD. In particular, via DPD, it is possible to model length scales up to $100 \mathrm{~nm}$ and time scales on the order of tens of microseconds. ${ }^{3}$ Below, we first detail our new DPD scheme. We then describe our simulations to capture the temperature-driven conformational changes of isolated fibers in solution that mimic the thermo-responsive behavior of the aptamers. Finally, we examine how the thermal response of the gel/fiber composite can be utilized for effective catch-and-release 
applications. As discussed below, the findings from these studies can be used to design microfluidic systems that trap and release biological cells, as well as nanofluidic devices that catch and release nanoparticles.

\section{Methodology}

\section{A. Details of the DPD simulation}

Our system involves an array of thermo-sensitive polymer fibers that adopt a hairpin structure at low temperature, $T$, and an unfolded chain structure at a higher $T$, with a critical temperature $T_{M}$ (see Figs. 1 and 2). The array of fibers is attached to a thermo-responsive hydrogel that exhibits a lower critical solution temperature (LCST) behavior, and thus, swells at $T<T_{c}$ and collapses at $T>T_{c}$. The gel/fiber composite is immersed in a fluid-filled chamber.

To model the dynamic behavior of this multi-component system, we utilize dissipative particle dynamics (DPD), ${ }^{3,7-8}$ which is a particle-based approach used to simulate the time evolution of a many-body system governed by Newton's equation of motion, $\mathrm{m} \mathrm{d} \mathbf{v}_{i} / \mathrm{d} t=\mathbf{f}_{i}$. Each bead $i$ in the system experiences a force $\mathbf{f}_{i}$, which is the sum of three pairwise additive forces: $\mathbf{f}_{i}(t)=\sum\left(\mathbf{F}_{i j}^{\mathrm{C}}+\mathbf{F}_{i j}^{\mathrm{D}}+\mathbf{F}_{i j}^{\mathrm{R}}\right)$, where the sum is over all beads $j$ within a certain cutoff radius $r_{\mathrm{c}}$ from bead $i$. The three forces are the conservative force $\mathbf{F}_{i j}^{\mathrm{C}}$, drag or dissipative force $\mathbf{F}_{i j}^{\mathrm{D}}$, and random force $\mathbf{F}_{i j}^{\mathrm{R}}$. We describe each pairwise force below.

The conservative force is a soft, repulsive force given by $\mathbf{F}_{i j}^{\mathrm{C}}=a_{i j}\left(1-r_{i j}\right) \hat{\mathbf{r}}_{i j}$, where $a_{i j}$ measures the maximum repulsion between beads $i$ and $j, r_{i j}=\left|\mathbf{r}_{i}-\mathbf{r}_{j}\right| / r_{\mathrm{c}}$, and $\hat{\mathbf{r}}_{i j}=\left(\mathbf{r}_{i}-\mathbf{r}_{j}\right) /\left|\mathbf{r}_{i}-\mathbf{r}_{j}\right|$. This soft-core force leads to a degree of overlap between neighboring beads and permits the use of larger time steps than those typically used in MD simulations, ${ }^{3}$ 
which commonly involve the use of hard-core potentials (e.g., the Lennard-Jones potential). The repulsive parameters $a_{i j}$ are given in terms of $\mathrm{k}_{\mathrm{B}} \mathrm{T}$. We choose room temperature as the reference value and thus $\mathrm{k}_{\mathrm{B}} \mathrm{T}_{0}=1$ with $T=25^{\circ} \mathrm{C}$. The reduced temperature is introduced as $T^{*}=T / T_{0}$, and hence, $T^{*}=1.077$ for $T=48^{\circ} \mathrm{C}$ and $T^{*}=1.01$ for $T=28^{\circ} \mathrm{C}$. All the repulsive parameters are listed in units of $T^{*}$ (see Table 1).

Table 1: List of interaction parameters in units of reduced temperature, $T^{*}$.

\begin{tabular}{|c|c|c|c|c|c|c|c|}
\hline & Solvent & Particle & Hairpin & $\begin{array}{c}\text { Unfolded } \\
\text { Fiber }\end{array}$ & $\begin{array}{c}\text { Top } \\
\text { Wall }\end{array}$ & $\begin{array}{c}\text { Bottom } \\
\text { Wall }\end{array}$ & $\begin{array}{c}\text { Polymer } \\
\text { Gel }\end{array}$ \\
\hline Solvent & 25 & 25 & 25 & 25 & 25 & 25 & $\chi_{\mathrm{ps}}$ \\
\hline Particle & - & 25 & 20 & 25 & 40 & 40 & 25 \\
\hline Hairpin & - & - & 25 & 25 & 40 & 40 & 40 \\
\hline Unfolded & & & & 25 & 40 & 40 & 40 \\
\hline Top Wall & - & - & - & & 25 & 25 & 25 \\
\hline Bottom & - & - & - & - & - & 25 & $\chi_{\mathrm{ps}}-8$ \\
\hline Polymer & - & - & - & - & & 25 \\
\hline
\end{tabular}

The drag force is $\mathbf{F}_{i j}^{\mathrm{D}}=-\gamma \omega_{\mathrm{D}}\left(r_{i j}\right)\left(\hat{\mathbf{r}}_{i j} \cdot \mathbf{v}_{i j}\right) \hat{\mathbf{r}}_{i j}$, where $\gamma$ is a simulation parameter related to viscosity arising from the interactions between beads, $\omega_{\mathrm{D}}$ is a weight function that goes to zero at $r_{\mathrm{c}}$, and $\mathbf{v}_{i j}=\mathbf{v}_{i}-\mathbf{v}_{j}$. The random force is $\mathbf{F}_{i j}^{\mathrm{R}}=\sigma \omega_{\mathrm{R}}\left(r_{i j}\right) \xi_{i j} \hat{\mathbf{r}}_{i j}$, where $\xi_{i j}$ is a zero-mean Gaussian random variable of unit variance and $\sigma^{2}=2 k_{\mathrm{B}} T \gamma$ relates the amplitude of the noise to the friction coefficient, as specified by the fluctuation-dissipation theorem. ${ }^{3,8}$ The value of $\gamma$ is chosen to ensure relatively rapid equilibration of the system's temperature and the numerical 
stability of the simulations for the specified time step. ${ }^{3}$ Finally, we use $\omega_{\mathrm{D}}\left(r_{i j}\right)=\omega_{\mathrm{R}}\left(r_{i j}\right)^{2}=\left(1-r_{i j}\right)^{2}$ for $r_{i j}<1.3,73$

Each of these three pairwise forces conserves momentum locally, and thus, DPD reproduces correct hydrodynamic behavior. ${ }^{3,7-8}$ The velocity-Verlet algorithm is applied to integrate the equations of motion in time. We take $r_{\mathrm{c}}$ as the characteristic length scale and $k_{\mathrm{B}} T$ as the characteristic energy scale in our simulations. The corresponding characteristic time scale is then defined as $\tau_{\text {intrinsic }}=\sqrt{m r_{\mathrm{c}}^{2} / k_{\mathrm{B}} T}$. The remaining simulation parameters are $\sigma=3$ and $\Delta t=0.02 \tau$, with a total bead number density of $\rho=3 .^{3}$

We take the initial configuration of the gel to be a finite-sized tetra-functional network with a diamond-like topology. ${ }^{5,9}$ The semi-flexible polymer strands are modeled as a sequence of 30 DPD beads that are connected by harmonic bonds, with an interaction potential given by $E=\frac{1}{2} K_{\text {bond }}\left(r-r_{0}^{g}\right)^{2}+K_{\text {angle }}(1+\cos \theta) \cdot{ }^{10-11}$ The first term in the latter expression characterizes the elastic energy with the elastic constant $K_{\text {bond }}$ and the second term represents the bending energy with the rigidity parameter $K_{\text {angle }}$. Here, $r_{0}^{g}=0.5$ is the equilibrium bond length and $\theta$ is the bond angle between two adjacent bonds. The bond and angle potentials of the gel are set respectively at $K_{\text {bond }}=128$ and $K_{\text {angle }}=4$; for these values, the ratio of the number of bond crossings to the total number of bonds is less than $2 \times 10^{-3}$ (as detailed in the SI), and the observed polymer concentration is comparable to the experimental results for this gel. ${ }^{12-13}$ Consequently, the total force acting on each gel bead is equal to $\mathbf{f}_{e}+\mathbf{f}_{i}$, where $\mathbf{f}_{e}=-\nabla E$ and $\mathbf{f}_{i}$ is the DPD pairwise force. 
As in our previous studies, ${ }^{10}$ the finite-sized network contains cross-links, which are beads with a connectivity of four, and dangling ends, which are located on the surface of the lattice and have a connectivity of less than four. Here, the gel contains 12,096 beads and consists of 396 strands, 175 cross-links, and 41 dangling ends. This network is periodic in the lateral ( $x$ and $y$ ) directions.

To properly model the thermo-responsive behavior of this gel, we relate the repulsive parameter between a polymer and solvent bead, $a_{\mathrm{ps}}$, to the Flory-Huggins parameter characterizing the polymer-solvent interaction, $\chi_{\mathrm{ps}}$, as follows: $a_{\mathrm{ps}}=a+k_{B} T \chi_{\mathrm{ps}} / 0.306 .^{3} \mathrm{In}$ studies of thermo-responsive gels (e.g., poly( $N$-isopropylacrylamide) (PNIPAAm)), it is typically assumed that $\chi_{\mathrm{ps}}$ depends on temperature and polymer concentration. ${ }^{6,14-16}$ Hence, we assume that $\chi_{\mathrm{ps}}(T, \varphi)=\chi_{1}(T)+\chi_{2} \varphi_{\mathrm{P}},{ }^{5}$ where $\varphi_{\mathrm{P}}$ is the polymer volume fraction in the gel and $\chi_{1}(T)=(\delta h-T \delta) / k_{B} T$, with $\delta h$ and $\delta$ seing the respective changes in enthalpy and entropy. ${ }^{6}$ ${ }^{17}$ Note that $\varphi_{\mathrm{P}}$ is calculated as $\varphi_{\mathrm{P}}=\bar{\rho}_{\mathrm{P}} / \bar{\rho}_{\text {gel }}$ where $\bar{\rho}_{\mathrm{P}}$ is the time-averaged polymer number density and $\bar{\rho}_{\text {gel }}$ is the time-averaged total number density of the gel including the polymer and solvent beads. Here, we set $a=25$ and take $\delta h=-14.331 \times 10^{-14} \mathrm{erg}, \delta s=-5.452 \times 10^{-16} \mathrm{erg} \cdot \mathrm{K}^{-1}$ and $\chi_{2}=0.596$ to produce a continuous volume transition between $T=30^{\circ} \mathrm{C}$ and $35^{\circ} \mathrm{C} .{ }^{3,18-22}$ With this choice of $a_{\mathrm{ps}}$, we reproduce the experimentally observed temperature-induced volume phase transitions of the PNIPAAm gels. ${ }^{5-6,23}$

Notably, in related studies involving polymer gels, we undertook a comparison between our DPD simulation approach and the corresponding experiments on the formation of "stackable" multilayer gels. ${ }^{24}$ In particular, we modeled gelation using atom transfer radical polymerization (ATRP) and successfully matched our simulation results to the experimental findings, and thereby, provided insight into the factors that affect the formation and binding of 
the different gel layers in the system. The observed correspondence between our simulation results and these experiments points to the accuracy of our approach.

The gel layer is attached via an adhesive interaction to the substrate (brown beads in Fig. 3a). The effective attraction between the gel and substrate is modeled by setting the interaction parameter between the beads in the gel and bottom wall, $a_{\mathrm{gw}}$, at $a_{\mathrm{gw}}=a_{\mathrm{ps}}-8$ so that the gel remains anchored to the wall in all our simulations.

The non-deformable spherical particle in Fig. 4 is constructed from 1,624 DPD beads that are dispersed on two spherical layers with an outer layer radius of 5 and interlayer spacing of 0.5 . Each spherical layer is modeled by geodesic grids generated by subdividing an icosahedron. ${ }^{25-27}$ In this way, we construct a particle with a well-defined smooth surface, with an outer diameter of 10. The total force and torque acting on this particle is computed as the sum of the forces and torques on its constituent DPD beads. The corresponding number density of the particle shell is 11.4, which is sufficiently high to prevent penetration of the polymer beads into the sphere and does not induce an unrealistic depletion force between the solvent beads ${ }^{28}$ (a behavior that can occur in particle-based simulation methods such as DPD). ${ }^{28}$ We note that in the model for the nanoparticle, the ratio between the rotational and translational moments of inertia is approximately equal to 20 and the diffusion coefficient of the particle is $D \approx 2.5 \times 10^{-4}$ (as detailed in the SI).

The thermo-responsive fiber is modeled as a flexible polymer chain connected by the harmonic bonds:

$$
E^{1-2}=\frac{1}{2} K_{\text {bond }}^{1-2} \sum_{i=0}^{N_{c}-1}\left(\left|\mathbf{R}_{i+1}-\mathbf{R}_{i}\right|-r_{0}\right)^{2},
$$


where $\mathbf{R}_{i}$ is the position vector of the $i$ th bead, $N_{c}=25$ is the number of beads in the chain and $r_{0}=1$. (This value of $r_{0}$ is equal to twice the equilibrium bond length in the gel and is close to the value used by Neimark et al. ${ }^{4}$ ). Here, $K_{\text {bond }}^{1-2}=200$ is an elastic constant describing the harmonic bond between the neighboring beads (e.g., beads "1" and "2"). To capture the conformational change of the chain such that it adopts the hairpin-like structure at $T<T_{M}$ and an ideal-chain structure at $T>T_{M}$, we introduce two additional types of bonds. The first is a harmonic bond connecting beads separated by two bonds (i.e., connecting next-neighbor beads along the chain, beads " 1 " and " 3 "):

$$
E^{1-3}=\frac{1}{2} K_{\text {bond }}^{1-3} \sum_{i=0}^{N_{c}-\mathbf{2}}\left(\left|\mathbf{R}_{i+2}-\mathbf{R}_{i}\right|-2 r_{0}\right)^{2},
$$

where the bond constant is set to $K_{\text {bond }}^{1-3}=320$. Second, we introduce a temperature-dependent Morse bond connecting beads separated by three or more bonds. The latter bond is described by the following Morse potential:

$$
E^{M}= \begin{cases}\frac{1}{2} K_{\text {bond }}^{M} \sum_{|i-j|>2}\left[\left(1-\mathrm{e}^{-\alpha\left(\mathbf{R}_{i}-\mathbf{R}_{j} \mid-r_{M}\right)}\right)^{2}-1\right], & T<T_{\mathrm{M}} \\ 0, & T>T_{\mathrm{M}}\end{cases}
$$

for $\left|\mathbf{R}_{i}-\mathbf{R}_{j}\right| \leq 1$. Here, $K_{\text {bond }}^{M}$ is the depth of the energy potential, $\alpha=8$ and its inverse value $\frac{1}{\alpha}$ is related to the width of the energy well. The equilibrium length of bonds is set to $r_{M}=0.5$. If the distance between two beads is greater than 1, the Morse potential is negligible since $E^{M} / K_{\text {bond }}^{M} \approx 3.6 \%$ and thus, we set the cutoff of the potential equal to 1 . This overall approach for modeling aptamers is based on DPD simulations of polypeptides in solution that successfully reproduced transitions among coil-like, globular, $\alpha$-helical, and $\beta$-hairpin configurations of 
model peptides. These simulations also yielded values for critical micelle concentrations that were in quantitative agreement with experimental measurements. ${ }^{4,29}$

Note that in eq. 1, the equilibrium distance between the bead and its next-nearest neighbor is equal to $2 r_{0}$. Therefore, the fiber, governed by the combination of $E^{1-2}$ and $E^{1-3}$, is in the energetically favored unfolded structure in the absence of $E^{M}$. The $E^{M}$ term alone, however, tends to bind the chain beads in more compact structures. As we discuss further below, the interplay of the three potentials is responsible for the final structure of the hairpin.

Variations in temperature affect not only the structure of aptamers, but also the binding affinity of these macromolecules. Namely, at $48^{\circ} \mathrm{C}$, unfolded aptamers were observed to release cells that were previously bound at $33^{\circ} \mathrm{C} .{ }^{2}$ We incorporate this behavior in our system by specifying that while the hairpin structure can bind the nanoparticle, the unfolded chain does not have an affinity to the particle. In particular, we set $a_{\mathrm{FP}}=20$ when the chain is in the hairpin conformation and $a_{\mathrm{FP}}=25$ when the chain is unfolded.

The $2 \times 2$ array of fibers is attached to the gel surface via a harmonic spring by connecting one end of each fiber to a gel bond. The inter-fiber spacing is initially equal to 13.2 along the $x$-direction and 8.2 along the $y$-direction. The top and bottom solid walls that bound the system in the $z$ direction are modeled as solid beads with a height $h=1$ and density $\rho_{\text {wall }}=3$. (The wall beads are organized in an amorphous arrangement.) Bounce-back boundary conditions are applied at the fluid-solid interfaces to prevent the solvent and gel beads from penetrating into the walls, and to produce no-slip boundary conditions with minimal interfacial density oscillations. ${ }^{11}$ Periodic boundary conditions are applied along the $x$ and $y$ directions. 
Shear is applied to the system by moving the upper wall of the simulation box along the $x$ direction at specified velocities. The shear rate $\dot{\gamma}$ takes the values $1.67 \times 10^{-4}, 1.67 \times 10^{-3}$, $8.33 \times 10^{-3}$, and $1.67 \times 10^{-2}$, which correspond respectively to the velocities of the upper wall of $0.01,0.1,0.5$, and 1 in dimensionless units.

Finally, the simulation box is $24.6 \times 24.6 \times 60$ units in size and is filled with 89,603 solvent beads, maintaining the total density of the system at $\rho_{\text {sys }}=3$. Eight independent simulations are carried out for $t=5 \times 10^{6}$ time steps for each parameter set. (In the ensuing discussion, $t$ is specified in simulation time steps, which can be related to physical units of time as discussed below.)

\section{B. Comparison of simulation parameters to physical values}

We can relate the dimensionless parameters to physical values through the value of the collective diffusion coefficient of the polymer network. If we assume that each solvent bead represents 10 water molecules, ${ }^{30-31}$ then a DPD solvent bead occupies a volume of $300 \mathrm{~A}^{3}$ since a water molecule (of mass density $1 \mathrm{~g} / \mathrm{cm}^{3}$ ), has a volume $\approx 30 \AA^{3}$. The total bead number density in our system is $\rho_{\text {sys }}=3$ and using $\rho_{\text {sys }}=3 r_{c}^{-3}$ and the mass density of water, we obtain the unit length $r_{c}=0.97 \mathrm{~nm}$ and the characteristic mass $m=180 \mathrm{Da}$. By matching the mass density of a polymer bead in the simulation to the mass density of amorphous PNIPAAm $\left(1.1 \mathrm{~g} / \mathrm{cm}^{3}\right)$, we find that a polymer bead represents 1.6 PNIPAAm monomers.

To obtain the correct characteristic time scale, we relate the collective diffusion coefficient of the polymer network in the simulations, $\mathrm{D}_{0}^{\mathrm{sim}}=1.74 \times 10^{-2} \mathrm{~nm}^{2} / \tau_{\mathrm{DPD}}$, obtained from the swelling kinetics of the gel, to the experimental value $\mathrm{D}_{0}^{\exp }=2 \times 10^{-11} \mathrm{~m}^{2} / \mathrm{s} .{ }^{32}$ We thus 
obtain the following physical values for the simulation parameters: $\tau_{\mathrm{DPD}}=0.87 \mathrm{~ns}$, the simulation box size is $23.9 \times 23.9 \times 58.2 \mathrm{~nm}$, the fiber equilibrium length is $27.2 \mathrm{~nm}$, and the particle diameter is $9.7 \mathrm{~nm}$. The values $K_{\text {bond }}=128$ and $K_{\text {angle }}=4$ correspond to $0.56 \mathrm{~N} / \mathrm{m}$ and $4 k_{B} T$, respectively. ${ }^{10}$ The diffusion coefficient of the nanoparticle is $D \approx 2.5 \times 10^{-4}$, which corresponds to the dimensional value of $D \approx 1.35 \times 10^{-11} \mathrm{~m}^{2} / \mathrm{s}$. The value of the applied shear rate ranges from $\dot{\gamma}=1.9 \times 10^{5} / \mathrm{s}$ to $\dot{\gamma}=1.9 \times 10^{7} / \mathrm{s}$; these shear rates are of the same order of magnitude as those used in high shear rate experiments in microfluidic devices. ${ }^{33-34}$ For these ranges of shear, the corresponding Peclet number can be roughly estimated as $P e \in[8,400]$ and hence, the motion of the nanoparticle is dominated by advection. ${ }^{35}$

\section{Results and Discussions}

In the ensuing discussion, we first focus on a single thermo-responsive fiber in solution to determine the optimal range of parameters that lead to the formation of a stable biomimetic, hairpin structure. We then anchor these fibers onto a swollen gel layer and examine the ability of the hairpin-forming fibers to trap nanoparticles, which are driven to flow over the gel layer in a channel. Finally, we investigate the effect of increasing the temperature of the system and thus, fully exploiting the thermo-responsive behavior of both the fiber and the gel in this application.

\section{A. Forming the hairpin structure from a single, free chain in solution}

Given the DPD fibers considered here, our aim is to mimic the conformational change in biological aptamers, which undergo a transition from a hairpin-like structure at $T<T_{\mathrm{M}}$ to an unfolded chain at $T>T_{\mathrm{M}}$. The value of the transition temperature for biological aptamers has been reported as $T_{\mathrm{M}}=33.5^{\circ} \mathrm{C}$ and recent observations indicate such aptamers are in the 
unfolded state at $48^{\circ} \mathrm{C}^{2}$ To drive an analogous conformational change in the fiber, we utilize the combination of three intra-molecular interaction potentials, $E^{1-2}, E^{1-3}$ and $E^{M}$ ((eqs. (1)(3)), and vary the bond strength $K_{\text {bond }}^{M}$ in the expression for $E^{M}$ (eq. (3)), which describes the temperature-dependent interaction between beads separated by three or more bonds. First, we focus on a single fiber within the simulation box in the absence of the polymer gel and fix the temperature at $T=28^{\circ} \mathrm{C}<T_{\mathrm{M}}$. Sixteen independent simulations for each set of parameters are carried out for $2 \times 10^{6}$ time steps, i.e., until the fiber has completely equilibrated to a stable conformation.

As $K_{\text {bond }}^{M}$ is varied in the range from 1 to 4 , the fiber can adopt the following three stable states: "chain", "hairpin", or "ring"; these structures are shown in the inset in Fig. 1a and are indicated by a yellow, red, or green dot, respectively. We distinguish among these distinct structures by calculating $N_{c}$, the number of contacts, which is determined by counting the number of Morse bonds formed between the beads in the same fiber. In particular, two beads within the same fiber are considered to be in contact if they are separated by: 1) three or more bonds, and 2) a distance that is less than the interaction radius between the beads (here set equal to 1). If $N_{c}=0$, then the fiber is in the "chain" state. If $0<N_{c}<20$, then the fiber is considered to be in the "hairpin" state and if $N_{c}>30$, then the fiber is in the "ring" state. (Recall the fiber is formed from 25 beads.)

Figure 1a reveals the behavior of $N_{c}$ as a function of $K_{\text {bond }}^{\prime \prime}$. As shown in the phase map (Fig.1b), two structures can coexist at a specific value of $K_{\text {bond }}^{M}$. For example, for $K_{\text {bond }}^{M}=1.5$, we observe the hairpin structure in two of the 16 simulations, while the remaining 14 
configurations are the unfolded chains. The average of $N_{c}$ is calculated using data only for the hairpin and ring structures; if we included the contribution from the unfolded chains, the lower bound of $N_{c}$ (after considering the statistical error) would be negative for the case of $K_{\text {bond }}^{M}=1.5$. A negative value, however, does not reflect the physical meaning of the contact number.

(a)

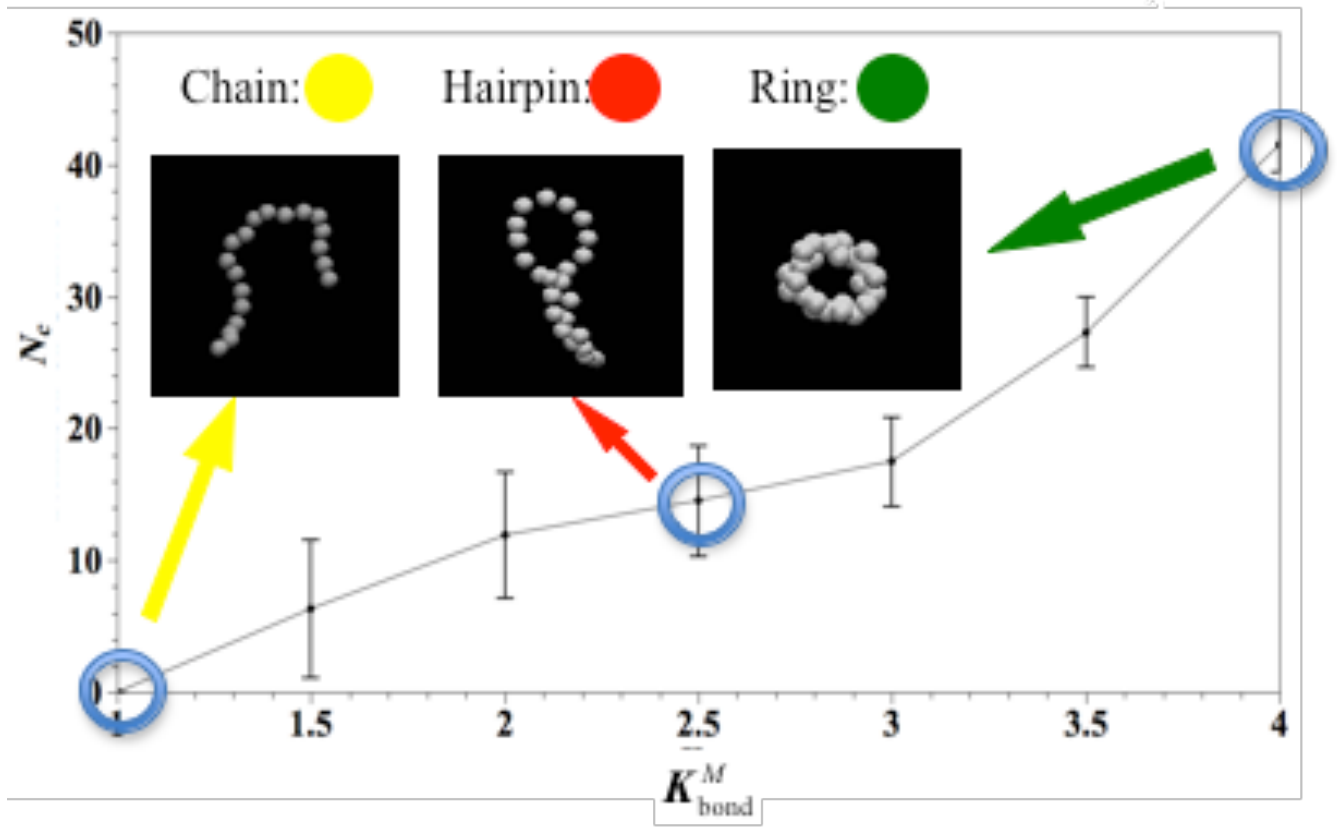

(b)

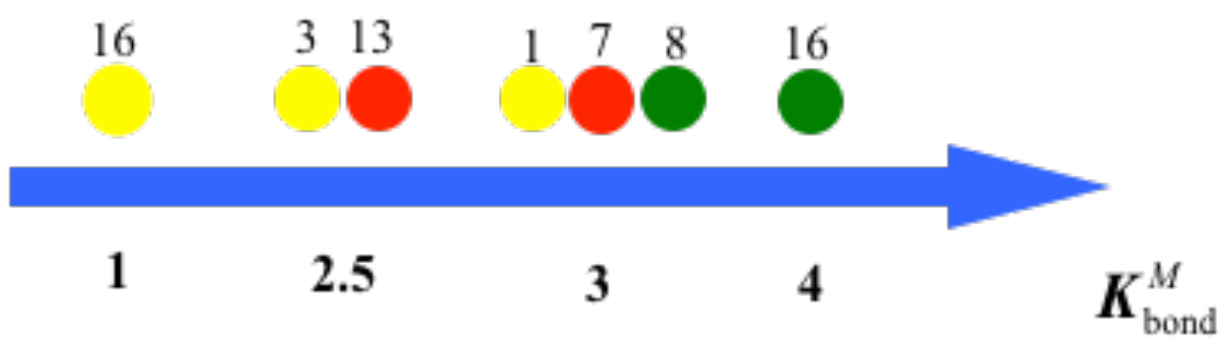

Figure 1. (a) Number of contacts, $N_{c}$, as a function of $K_{\text {bond }}^{M}$. Each error bar in the plot corresponds to the standard deviations obtained from 16 independent simulations. Images in the inset show the three types of observed structures: chain (marked by the yellow circle), hairpin (red circle), and globule (green circle). (b) Phase map of the observed structures as a function of $K_{\text {bond }}^{M}$. Values associated with each circle represent the number of occurrences of that structure in 16 independent simulations. 
Taken together, Figs. 1a and $1 \mathrm{~b}$ reveal the optimal conditions for achieving the different fiber conformations. For the weakest bonding interaction, $K_{\text {bond }}^{M}=1$, we find $N_{c}=0$; the latter value is consistent with the data in the phase map, where the unfolded chains are present in all 16 simulations. The radius of gyration of these chains is found to be $R_{g}=5.3 \pm 0.98$. Notably, when $K_{\text {bond }}^{M}$ is small, the motion of the fiber is dominated by $E^{1-2}$ and $E^{1-3}$, which both favor the unfolded conformation since the equilibrium distance between the bead and its next-nearest neighbor is equal to $2 r_{0}$ in the absence of $E^{M}$.

As $K_{\text {bond }}^{M}$ is increased to 1.5 , we begin to observe the presence of the hairpin structure. The error bars for $N_{c}$ are relatively large (Fig. 1a) at this value of $K_{\text {bond }}^{M}$ because thermal fluctuations can break this weak Morse bond. As $K_{\text {bond }}^{M}$ is increased further, we find that $K_{\text {bond }}^{M}=2.5$ constitutes the optimal value for forming the hairpin structure. Namely, the hairpin structure is formed in 13 of the 16 independent simulations. The radius of gyration of these hairpin chains is equal to $R_{g}=3.2 \pm 0.1$. In this case, the value of $E^{M}$ (the term that favors the compact structure) is comparable to the sum of $E^{1-2}$ and $E^{1-3}$.

When $K_{\text {bond }}^{M}=3$, the stronger Morse bond compresses the fiber into a more compact ring structure and this leads to a significant increase in the value of $N_{c}$. For the 16 independent simulations at $K_{\text {bond }}^{M}=3$, we obtained one "chain", seven "hairpins" and eight "ring" structures. When $K_{\text {bond }}^{M}$ is increased to 3.5 , there are no fibers in the "chain" state, while four are in the "hairpin" and 12 are in the "ring" state. As the Morse bonding potential is increased to $K_{\text {bond }}^{M}=4$, the fiber assumes the ring structure for all 16 simulations and $R_{g}=1.3 \pm 0.05$. 
Fig. 2 shows the typical kinetic pathway to forming the hairpin structure; here, we have fixed $K_{\text {bond }}^{M}=2.5$. The temporal evolution of $N_{c}$ (black curve) and $R_{g}$ (red curve) consistently display three distinct stages (see Fig. 2), where regions I, II, and III encompass the respective conformations shown below the plot. In stage I, $N_{c}=0$ and $R_{g}$ is close to the value of unfolded chains. At these early times, the fiber has not yet become sufficiently bent to form the Morse bonds. The duration of stage I is related to the diffusive motion of the fiber beads.

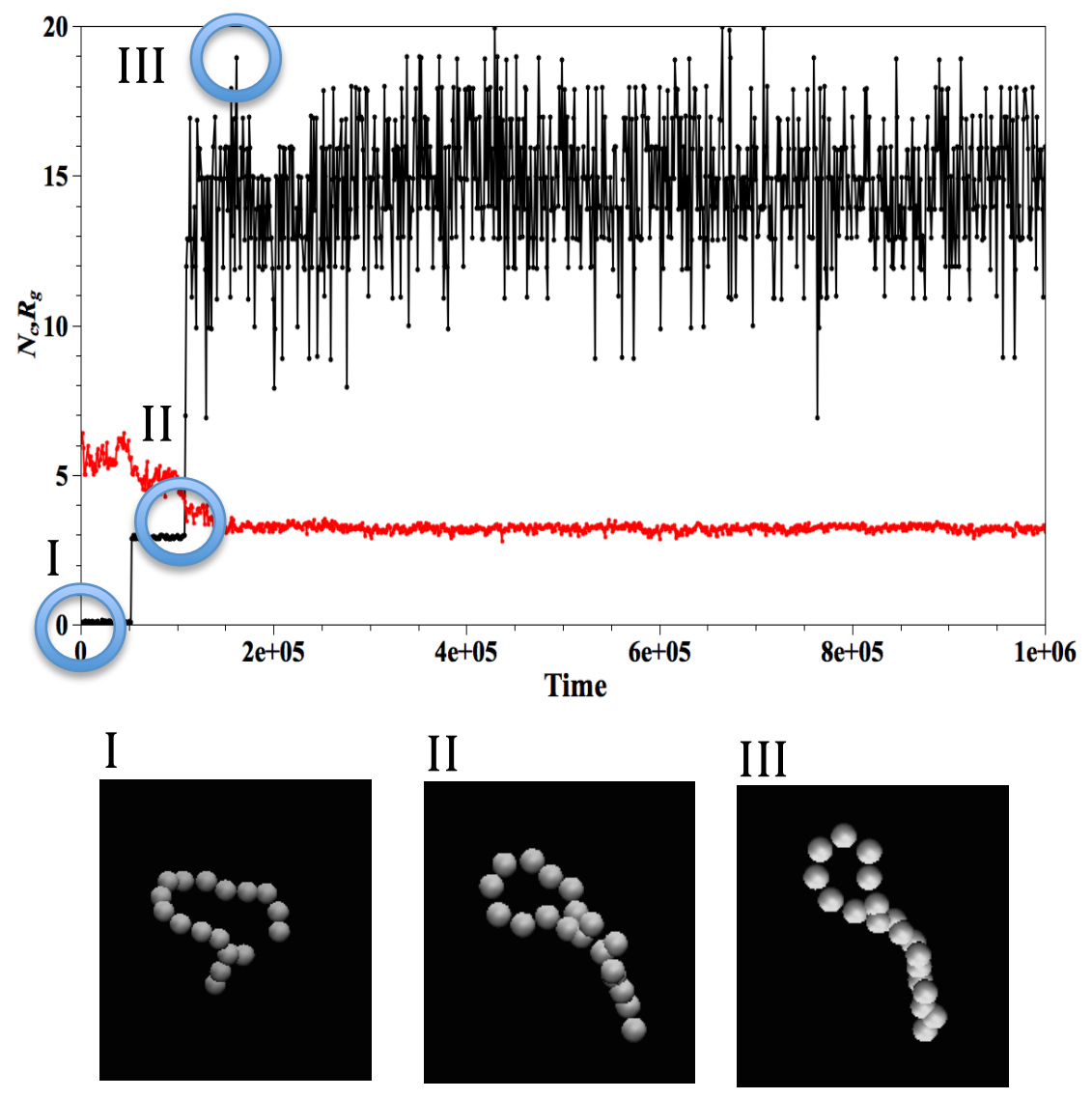

Figure 2. Typical kinetic pathway involved in forming a hairpin. Here, we set $K_{\text {bond }}^{M}=2.5$. Black curve indicates the number of contacts, $N_{c}$, that form as the fiber undergoes a transition from a chain (see image marked I) via an intermediate structure (see image marked II) to a hairpin (see inset marked III). Red curve corresponds to the radius of gyration, $R_{g}$, of the fiber. 
Once the fiber beads come into sufficiently close contact to form strong Morse bonds, the system enters stage II, where the fiber bends to a greater degree to increase the number of enthalpically favorable contacts. Consequently, the fiber displays a decrease in $R_{g}$.

In stage III, as more beads are bonded through $E^{\prime \prime}$ (which drives contacts between collinear beads separated by three or more bonds), the fiber forms a hairpin. The central loop in the hairpin exhibits relatively large curvature, which in turn affects the degree of stretching between nearest and next nearest neighbor beads. Hence, the energetic contributions from the $E^{1-2}$ and $E^{1-3}$ now begin to play a significant role. In other words, the interplay among the three potentials leads to a stable hairpin structure. Notice that in stage III, $N_{c}$ displays large fluctuations compared to $R_{g}$ since a single fiber bead can have multiple contacts, and thus, a small change of conformation $\left(R_{g}\right)$ can lead to large variations in $N_{c}$ (even if $R_{g}$ remains relatively constant).

\section{B. Catching a nanoparticle with hairpins anchored to a swollen gel at fixed temperature}

Having pinpointed $K_{\text {bond }}^{M}=2.5$ as the optimal value for driving the chain to form a hairpin, we investigate the system shown in Fig. 3. At the outset of the simulations, the polymer gel is equilibrated at the temperature $T=28^{\circ} \mathrm{C}$ and the array of four thermo-sensitive fibers are aligned along the $z$-direction (Fig. 3a). The gel is assumed to be PNIPAAM, which displays LCST behavior and thus, the gel is swollen at this low temperature.

Fig. $3 \mathrm{~b}$ shows the configuration of the system after it was equilibrated for $2 \times 10^{6}$ time steps at the same temperature $\left(T=28^{\circ} \mathrm{C}\right)$ to allow for the fibers to reach their equilibrium conformation; since the fibers are incompatible with the gel $\left(a_{i j}=40\right)$, the hairpins extend away from the gel layer and into the solution. We investigate how to utilize this system to extract a 

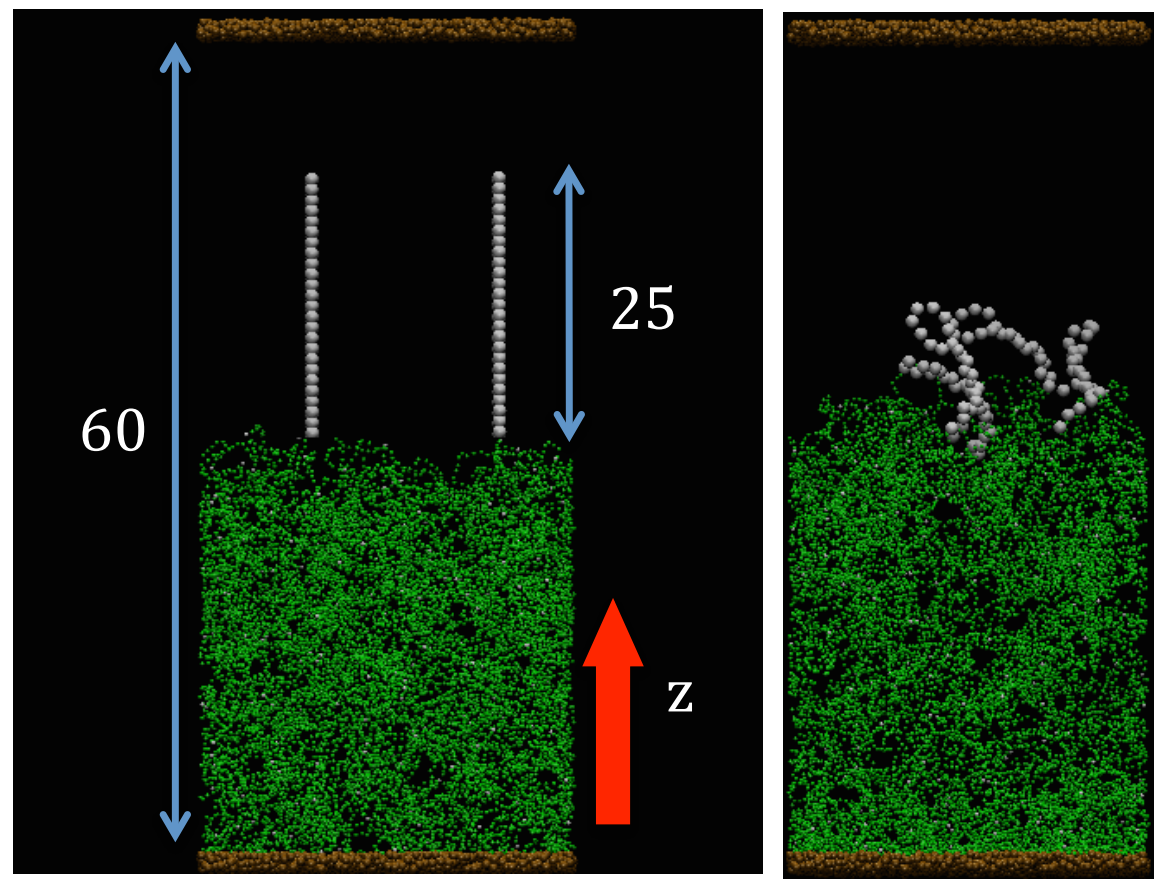

Figure 3. (a) Initial morphology of the system. (b) Morphology of the system at $t=10^{6}$ after equilibration at temperature equal to $T=28^{\circ} \mathrm{C}$.

targeted nanoparticle from the bulk of the solution at $T=28^{\circ} \mathrm{C}$. As shown in Fig. 4a, a particle with a diameter of 10 is introduced into the simulation box. At this initial stage, there is no point of contact between the particle and the gel or the hairpins; in fact, the minimum separation between the particle and hairpin is greater than four. We apply a shear flow (from the left to right of the simulation box along the $x$-direction) with the rate $\dot{\gamma}=1.67 \times 10^{-3}$ to propel the particle through the system.

As shown in Fig 4b, the hairpins begin interacting with the particle at $t=4 \times 10^{4}$ for this simulation, with the system encompassing a few hairpin-particle contacts. The snapshot of the system at $t=1.1 \times 10^{6}$ (Fig. $4 \mathrm{c}$ ) shows that at late times, all four hairpins are wrapped around the particle, causing the particle to be arrested on the surface and trapped in that position by a large number of hairpin-particle contacts. Due to the applied shear flow, one hairpin is significantly stretched, but is nonetheless attached to the particle. Notably, the swelling of the gel 
drives the hairpins close to the top of the chamber and into the path of the fluid-driven particle (see SI), and in this way, the thermo-responsive behavior of this LCST gel aids in the trapping of the particle.

(a)

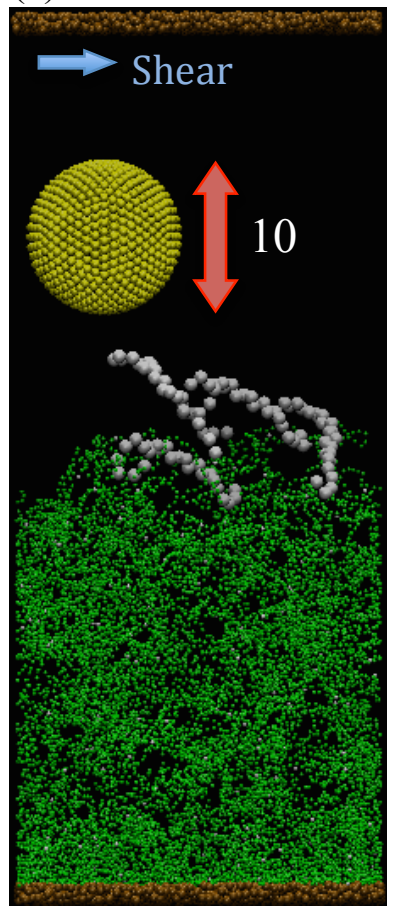

(b)

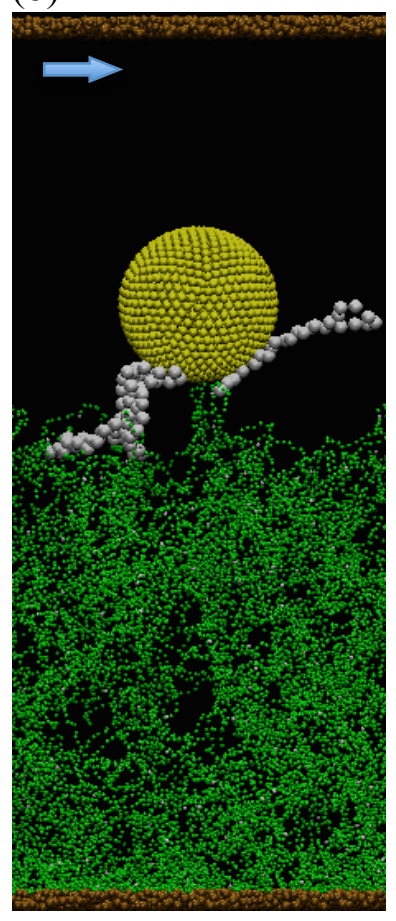

(c)

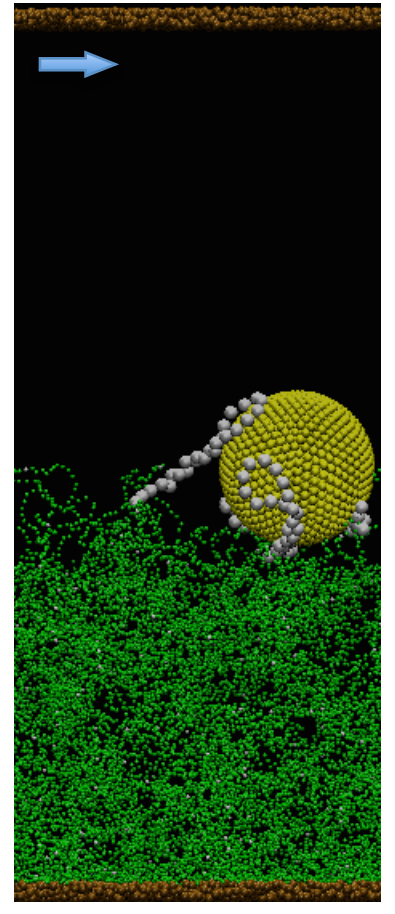

Figure 4. (a) Initial morphology of the system after equilibration in the imposed flow for $5 \times 10^{3}$ time steps. (b), (c) Snapshots of the system at $t=4 \times 10^{4}$ and $1.1 \times 10^{6}$, respectively, for $\dot{\gamma}=1.67 \times 10^{-3}$.

The rate of the imposed shear can affect the ability of the hairpins to catch and retain the particle. To probe the functionality of the system at different shear rates, we characterize the behavior of both the particle and hairpins (Fig. 5) at the following shear rates: $1.67 \times 10^{-4}$ (blue curve), $\quad 1.67 \times 10^{-3}$ (green curve), $8.33 \times 10^{-3}$ (red curve), and $1.67 \times 10^{-2}$ (black curve). We first monitor the velocity of the particle as a function of time, averaging over eight independent simulations that were carried out for $5 \times 10^{6}$ time steps. The shading about each solid line corresponds to the standard deviations from these simulations. For the relatively weak shears 
(a)

(b)
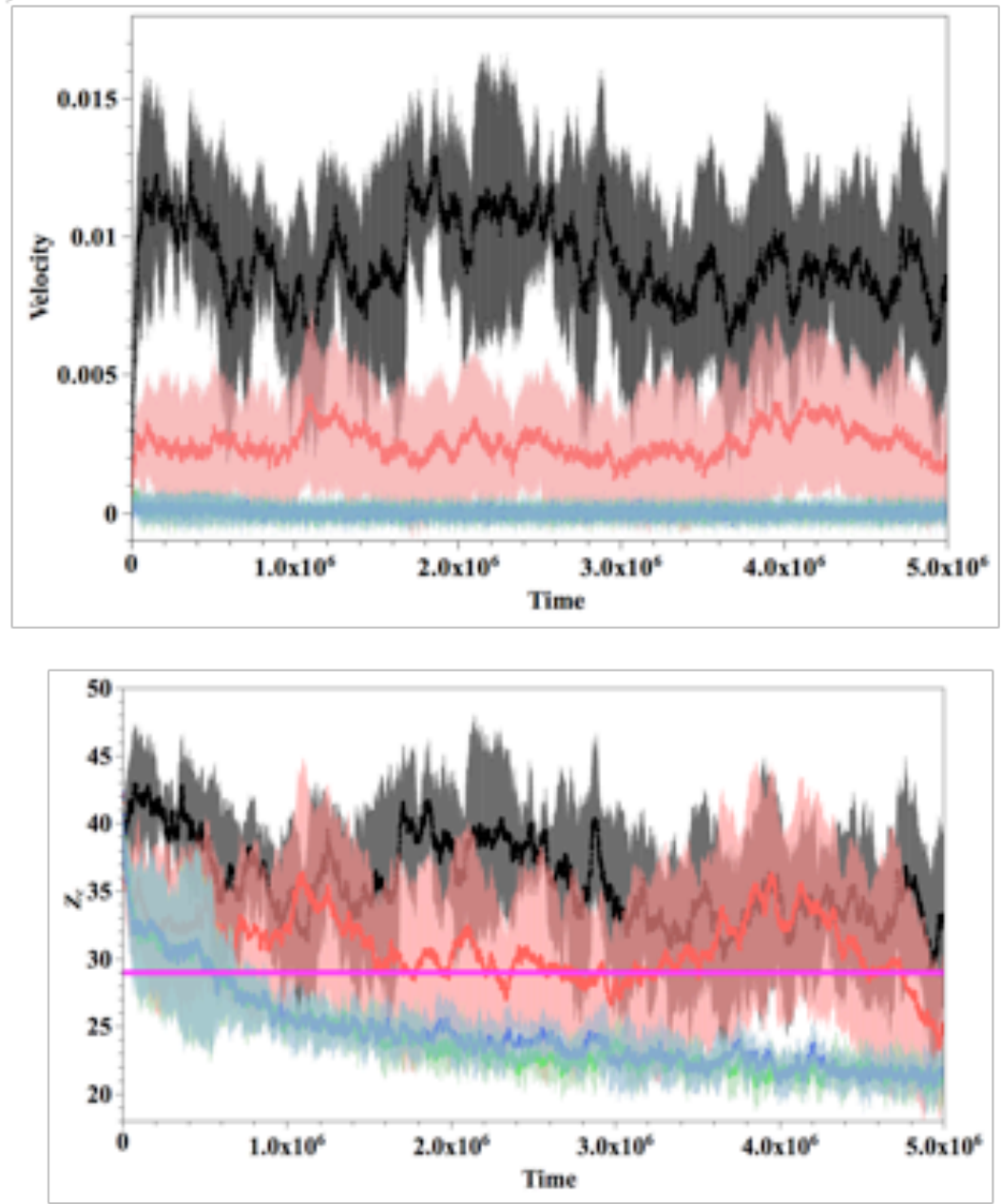

(c)

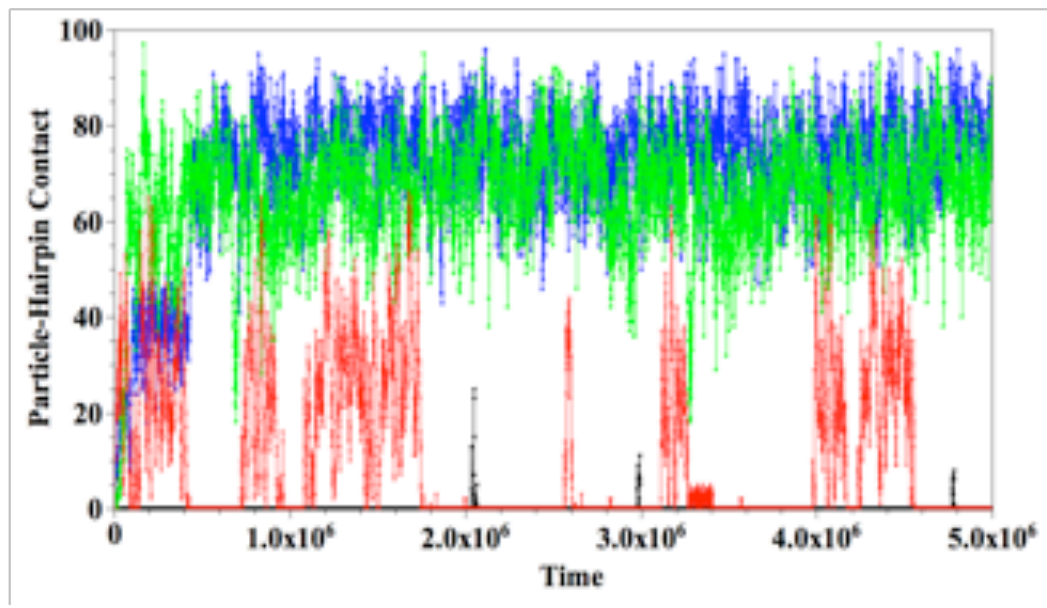

Figure 5. (a) Temporal evolution of the particle velocity along the flow direction for the following imposed shear velocities: $\dot{\gamma}=1.67 \times 10^{-4}$ (blue), $1.67 \times 10^{-3}$ (green), $8.33 \times 10^{-3}$ (red), and $1.67 \times 10^{-2}$ (black). (b) Corresponding temporal evolution of $Z_{c}$; magenta line is the height of the gel interface. (c) Corresponding temporal evolution of the number of particle- 
hairpin contacts.

$\dot{\gamma}=1.67 \times 10^{-4}$ and $1.67 \times 10^{-3}$, the average particle velocities are close to zero; note that both curves (blue and green) overlap and the standard deviations are small (Fig. 5a). These findings suggest that at relatively low shear rates, the particle is caught and its motion becomes arrested quite rapidly, as indicated by the images in Fig. 4.

As the shear rate is increased to the moderate value of $\dot{\gamma}=8.33 \times 10^{-3}$, the particle velocity is greater than zero, but is still relatively small (compared to the black curve). In this case, the shear force acting on the particle is comparable to the binding force induced by the hairpin, and thus, the particle velocity is decreased by its interaction with the fiber. Namely, the shear-driven velocity of the particle in the absence of the fibers is equal to $6 \times 10^{-3}$ and is equal to $2.5 \times 10^{-3}$ in the presence of the hairpins. As the particle is driven across the simulation box, it attaches to and detaches from the fibers multiple times.

When the shear is increased to the highest value considered here, $\dot{\gamma}=1.67 \times 10^{-2}$, the force due to the adhesion between the hairpin and the particle is weaker than the shear force and consequently, the motion of the particle is mainly unaffected by the fibers (a relatively small number of contacts that are created between the particle and fibers are almost immediately broken by the strong shear force, as can be seen from the black line in Fig. 5c).

To better understand the competition between the action of the hairpin and the imposed shear on the motion of the particle, we monitor the temporal evolution of $Z_{c} \mathrm{Z}_{\mathrm{c}}$, the vertical position of the bottom of the particle, as shown in Fig. 5b. As a point of reference, the height of the gel/fluid interface, $h_{\text {gel }} h_{\text {gel }}$, is approximated as $h_{\text {gel }}=\left\langle Z_{\text {gel }}\right\rangle-1$; here, $\left\langle Z_{\text {gel }}\right\rangle$ is the center of 
mass of the gel. We calculate $\left\langle Z_{\text {gel }}\right\rangle$ by averaging over the $z$ coordinates of all the gel beads (and subtract 1 since the gel is attached to the bottom wall of height of 1 ). We obtain $h_{\text {gel }}=29 \pm 1.2$ (drawn in magenta in Fig. 5b) and neglect the fluctuations in the plot. In the case of the weak shears (blue and green curves), the particle position is seen to rapidly and consistently decrease as the particle approaches the gel surface; the small fluctuations about the curves indicate that the particle is firmly bound to the hairpins.

For the strongest shear, $\dot{\gamma}=1.67 \times 10^{-2}, Z_{c}>h_{\text {gel }}$ and the particle is not caught by the hairpins. For the intermediate value of $\dot{\gamma}=8.33 \times 10^{-3}$, the red curve displays large fluctuations and exhibits characteristics of both the weak and strong shear regimes. In this intermediate regime, the hairpin still interacts with the particle, forming a few numbers of contacts, as in the case of weak shear. Since the shear force is comparable to the relatively weak adhesive interaction, the particle repeatedly binds to and unbinds from the fibers as it passes over the surface.

The above arguments are supported by the time evolution of the particle-hairpin contacts, as shown in Fig. 5c. The blue and green curves, which correspond to the weak shears, exhibit a sharp increase and reach a saturation value of about 80 . This value corresponds to the situation in Fig. 4c, where four hairpins are wrapped around the particle. The particle-hairpin contact for the moderate shear displays large variations (red curve), which indicates that the particle and hairpin come into contact frequently, but this binding interaction is broken by the imposed flow (points where the contact equals zero). For the strongest shear, the number of particle-hairpin contacts remains essentially equal zero, consistent with the observation in Fig. 5a and 5b.

\section{Increasing the temperature to drive the release of the particle}


As noted above, the hairpin fibers mimic the properties of aptamers that unfold above a critical temperature, $T_{M}$. Before investigating the effect of increasing the temperature on the entire gel/fiber composite, we first investigate the unfolding dynamics of a single fiber. Recall that the bond connecting beads separated by three or more bonds is temperature-dependent (eq. (3)). Namely, $E^{M}=0$ when $T>T_{\mathrm{M}}$. Consequently, at $T>T_{\mathrm{M}}$, the conformation of the fiber is governed by the combination of $E^{1-2}$ and $E^{1-3}$; these terms drive the chain to adopt a more extended, unfolded structure. As shown in Fig. 6, after we increase the system temperature above the transition temperature by setting $T=48^{\circ} \mathrm{C}, N_{c}$ decreases to 16 at $t=2 \times 10^{3}$, producing the conformation shown in inset 1 . At $t=10^{4}, N_{c}=2$ and most of the fiber beads are no longer in contact (as shown in inset 2). Insets 3 and 4 correspond to $t=5 \times 10^{4}$ and $6 \times 10^{4}$, respectively, and the fiber transforms from the hairpin structure to an unfolded chain where the corresponding $N_{c}=0$.

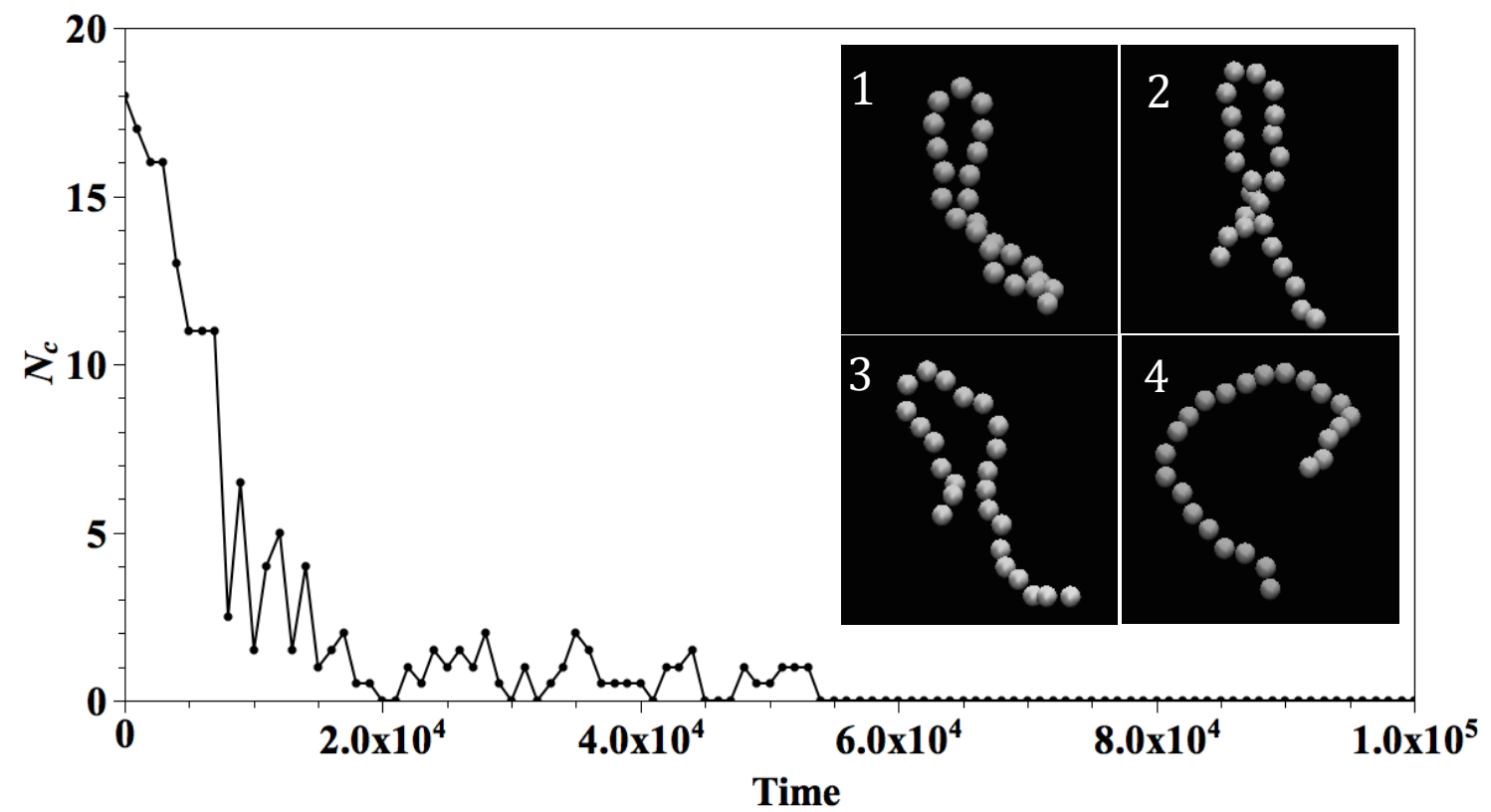

Figure 6. Dynamic pathway for the unfolding of the hairpin at $t=2 \times 10^{3}(1), 10^{4}$ (2), $5 \times 10^{4}(3)$, and $6 \times 10^{4}(4)$. Here, we set $K_{\text {bond }}^{M}=2.5$ and $T=48^{\circ} \mathrm{C}$. 
For the aptamers, the unfolding of the chain leads to a loss of biological activity, i.e., an inability to bind the targeted molecules. In our simulations, we mimic this behavior by increasing the repulsion between a fiber and a particle bead when the chain is unfolded. Hence, when the hairpin unfolds, a bound particle can readily detach from the fiber and be removed by the imposed the shear flow. We exploit this behavior in our gel/fiber composite, as well as taking advantage of thermo-responsive shrinking of the gel.

In the following simulations, we consider the behavior of the entire gel/fiber composite system when the temperature of the system is increased above the critical temperature $T_{\mathrm{M}}$. We begin with the system at $T=28^{\circ} \mathrm{C}$ and focus on the scenario where the particle is bound by the hairpins, as shown in Fig. 7a. Here, a shear of rate of $\dot{\gamma}=1.67 \times 10^{-3}$ is applied along the $x$ direction. To illustrate the merit of utilizing the thermo-responsive gel in the composite, we now

(a)

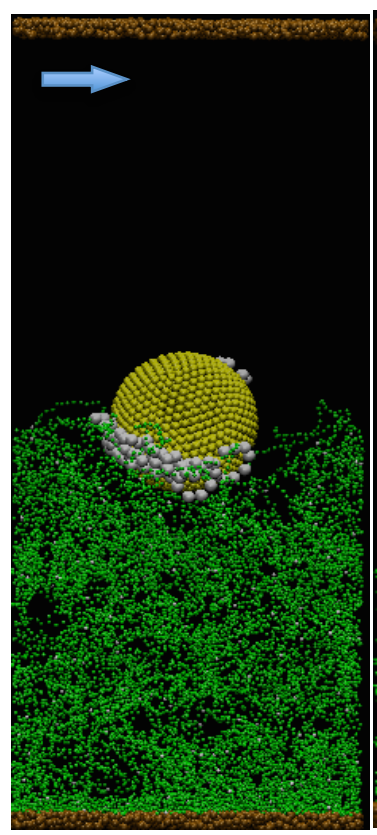

(b)

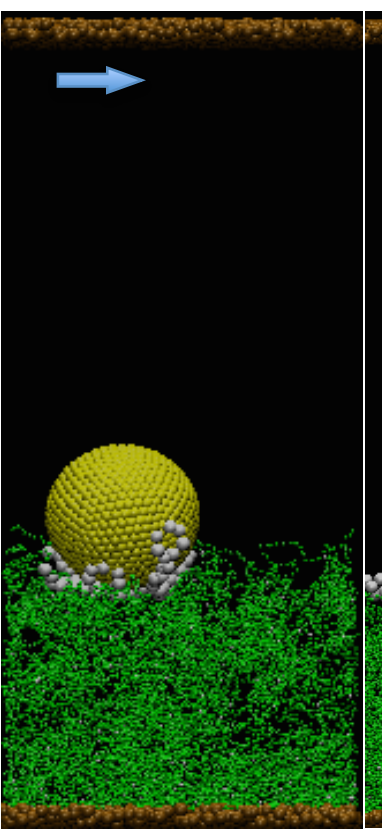

(c)

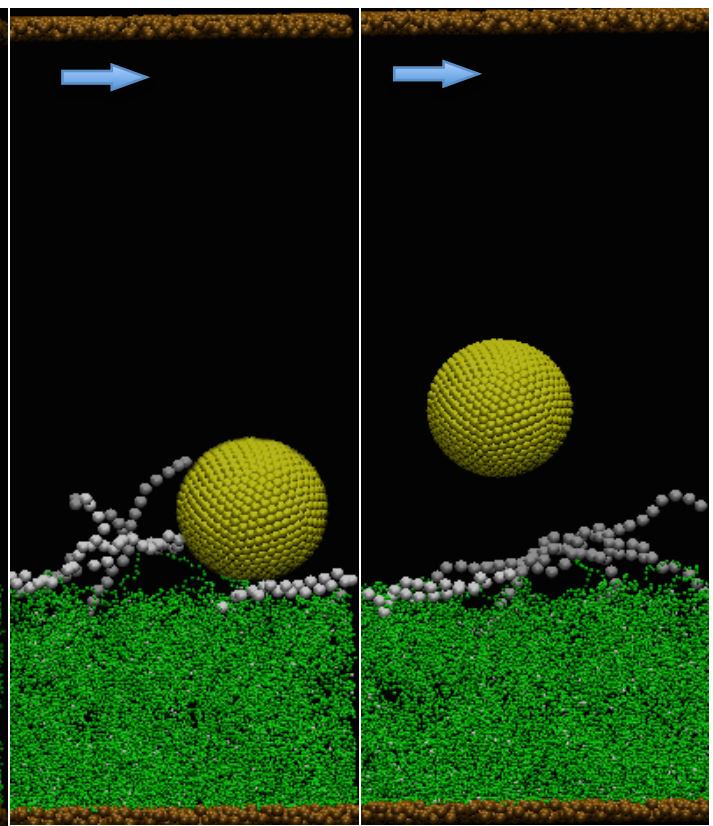

Figure 7. (a) Initial morphology of the system at the low temperature of $T=28^{\circ} \mathrm{C}$. (b) Snapshot of the system at $t=3 \times 10^{6}$ after the temperature was increased to $T=32^{\circ} \mathrm{C}$. The 
gel shrinks while the particle is arrested by the hairpin. (c) System with the morphology shown in (b) is heated to $T=40^{\circ} \mathrm{C}$ and the snapshot corresponds to $t=3.024 \times 10^{6}$ under the shear rate $\dot{\gamma}=8.33 \times 10^{-3}$. (d) Morphology of the system at $t=3.188 \times 10^{6}$.

employ a two-step process to increase the temperature in the system. In the first step, the temperature of the system is increased to $T=32^{\circ} \mathrm{C}<T_{M}$. At this temperature, the thermoresponsive LCST gel shrinks, but the fiber remains in the hairpin structure. Hence, the particle remains bound to the fibers and is drawn downward toward the substrate by the collapsing gel, as illustrated in Fig. 7b, which shows a snapshot of the system at $t=3 \times 10^{6}$. (By comparing Figs. $7 \mathrm{a}$ and $7 \mathrm{~b}$, one can clearly see that the height of the gel has decreased at the higher temperature.)

In the second stage of the heating process, the temperature is further increased to above the transition temperature $T_{M}$. Therefore, the hairpins unfold (see Fig. 7d) and simultaneously, the fibers lose their affinity to the particle. Due to the applied shear, the fibers are stretched along the shear direction. Notably, the particle has detached from the fibers and gel interface, and is completely released to the bulk solution (Fig. 7d). ${ }^{36}$ The shrinking of the thermo-responsive gel plays an important role because it increases the unobstructed volume that is available for the flowing fluid, which transports the particle away from the layer.

Importantly, the behavior of the system can be reset by decreasing the temperature. In particular, the gel expands and the chains refold into the hairpin structure as temperature is decreased to $T=28^{\circ} \mathrm{C}$. (The conformation and activity of certain apatmers can be recovered through a decrease in temperature. ${ }^{2}$ ) Hence, the temperature of the system can be cycled to promote the trapping of the particles at low temperature and their release at high temperature.

\section{Conclusions}


Using computational modeling, we designed an effective "catch-and-release" system where the components of a thermo-responsive gel/fiber composite act in concert to extract nanoparticles in solution at low temperatures and release the particles at a higher temperature. To perform these studies, we devised a new DPD model that integrates the thermo-responsive behavior of both the bio-inspired fibers and LCST gel. Thus, we could probe and visualize the mechanisms that gave rise to this useful functionality.

In this system, the thermo-responsive fiber can be made to reversibly bind and unbind the targeted nanoparticles by varying the temperature, which induces the conformational changes of this chain. In contrast to previous devices that exploit the conformational changes of aptamers for "catch-and-release" applications, ${ }^{2}$ the system described here harnesses a substrate that is coated with a LCST gel (for example, PNIPAAm). The advantageous feature of this system is that the swelling of gel brings the anchored hairpins closer to the top of the chamber and hence, into the path of the fluid-driven particles, thereby facilitating the "catch" part of the process. Moreover, when the temperature is increased, the gel collapses and thus, effectively increases the space that can be occupied by the imposed flow, which is utilized to detach the particle from the unfolded chain and transport the freed particle within the device. Since the temperature-induced conformational changes of the polymer and fiber are reversible, the system can be used to repeatedly catch and release the particles.

Our results revealed a range of shear rates where the fiber can trap the particles, as well as the flow rates where the particles can be released to the surrounding solution. Notably, the system also provides a useful platform for purification and filtration applications, where it is important to prevent the extracted particles from re-entering the fluid. At $T=28^{\circ} \mathrm{C}$, the system could provide exactly that function. Once the purified fluid has been extracted from the chamber, 
a new fluid could be introduced into the device and the temperature could be increased to dislodge the trapped particles. By flushing these particles from the system and then lowering the temperature, the layer could again be used as a purification system.

\section{Acknowledgements}

This work was supported by Department of Energy under the Award number DE-SC0005247.

\section{References}

1. Shastri, A.; McGregor, L. M.; Liu, Y.; Harris, V.; Nan, H.; Mujica, M.; Vasquez, Y.; Bhattacharya, A.; Ma, Y.; Aizenberg, M.; Kuksenok, O.; Balazs, A. C.; Aizenberg, J.; He, X., An Aptamer-Functionalized Chemomechanically Modulated Biomolecule Catch-and-Release System. Nat. Chem. 2015, 7 (5), 447-454.

2. Zhu, J.; Nguyen, T.; Pei, R.; Stojanovic, M.; Lin, Q., Specific Capture and TemperatureMediated Release of Cells in an Aptamer-Based Microfluidic Device. Lab Chip 2012, 12 (18), 3504-3513.

3. Groot, R. D.; Warren, P. B., Dissipative Particle Dynamics: Bridging the Gap between Atomistic and Mesoscopic Simulation. J. Chem. Phys. 1997, 107 (11), 4423-4435.

4. Vishnyakov, A.; Talaga, D. S.; Neimark, A. V., DPD Simulation of Protein Conformations: From alpha-Helices to beta-Structures. J. Phys. Chem. Lett. 2012, 3 (21), 30813087.

5. Yong, X.; Kuksenok, O.; Matyjaszewski, K.; Balazs, A. C., Harnessing InterfaciallyActive Nanorods to Regenerate Severed Polymer Gels. Nano Lett. 2013, 13 (12), 6269-6274.

6. Hirotsu, S., Softening of Bulk Modulus and Negative Poisson's Ratio near the Volume Phase Transition of Polymer Gels. J. Chem. Phys. 1991, 94 (5), 3949.

7. Hoogerbrugge, P. J.; Koelman, J. M. V. A., Simulating Microscopic Hydrodynamic 
Phenomena with Dissipative Particle Dynamics. EPL 1992, 19 (3), 155-160.

8. Español, P.; Warren, P., Statistical Mechanics of Dissipative Particle Dynamics. EPL 1995, 30 (4), 191-196.

9. Jha, P. K.; Zwanikken, J. W.; Detcheverry, F. A.; de Pablo, J. J.; de la Cruz, M. O., Study of Volume Phase Transitions in Polymeric Nanogels by Theoretically Informed Coarse-grained Simulations. Soft Matter 2011, 7 (13), 5965-5975.

10. Liu, Y.; McFarlin, G. T. t.; Yong, X.; Kuksenok, O.; Balazs, A. C., Designing Composite Coatings That Provide a Dual Defense against Fouling. Langmuir 2015, 31 (27), 7524-7532.

11. Pan, W.; Fedosov, D. A.; Karniadakis, G. E.; Caswell, B., Hydrodynamic Interactions for Single Dissipative-Particle-Dynamics Particles and Their Clusters and Filaments. Phys. Rev. E 2008, $78(4), 046706$.

12. Nikunen, P.; Vattulainen, I.; Karttunen, M., Reptational Dynamics in Dissipative Particle Dynamics Simulations of Polymer Melts. Phys. Rev. E 2007, 75 (3), 036713.

13. Karatrantos, A.; Clarke, N.; Composto, R. J.; Winey, K. I., Topological Entanglement Length in Polymer Melts and Nanocomposites by a DPD Polymer Model. Soft Matter 2013, 9 (14), 3877-3884.

14. Quesada-Pérez, M.; Maroto-Centeno, J. A.; Forcada, J.; Hidalgo-Alvarez, R., Gel Swelling Theories: the Classical Formalism and Recent Approaches. Soft Matter 2011, 7 (22), 10536-10547.

15. Hirotsu, S., Phase-Transition of a Polymer Gel in Pure and Mixed-Solvent Media. J. Phys. Soc. Jpn. 1987, 56 (1), 233-242.

16. Erman, B.; Flory, P. J., Critical Phenomena and Transitions in Swollen Polymer Networks and in Linear Macromolecules. Macromolecules 1986, 19 (9), 2342-2353. 
17. Flory, P. J., Principles of Polymer Chemistry. Cornell University Press: Ithaca, NY, 1953.

18. Chang, H. Y.; Lin, Y. L.; Sheng, Y. J., Multilayered Polymersome Formed by Amphiphilic Asymmetric Macromolecular Brushes. Macromolecules 2012, 45 (11), 4778-4789.

19. Groot, R. D.; Madden, T. J., Dynamic Simulation of Diblock Copolymer Microphase Separation. J. Chem. Phys. 1998, 108 (20), 8713.

20. Hellweg, T.; Dewhurst, C. D.; Bruckner, E.; Kratz, K.; Eimer, W., Colloidal Crystals Made of Poly(N-isopropylacrylamide) Microgel Particles. Colloid. Polym. Sci. 2000, 278 (10), $972-978$.

21. Guo, J.; Liang, H.; Wang, Z. G., Coil-to-Globule Transition by Dissipative Particle Dynamics Simulation. J. Chem. Phys. 2011, 134 (24), 244904.

22. Soto-Figueroa, C.; Rodriguez-Hidalgo, M. D.; Vicente, L., Dissipative Particle Dynamics Simulation of the Micellization-Demicellization Process and Micellar Shuttle of a Diblock Copolymer in a Biphasic System (Water/Ionic-Liquid). Soft Matter 2012, 8 (6), 1871-1877.

23. Schild, H. G., Poly (N-Isopropylacrylamide) - Experiment, Theory and Application. Prog. Polym. Sci. 1992, 17 (2), 163-249.

24. Yong, X.; Simakova, A.; Averick, S.; Gutierrez, J.; Kuksenok, O.; Balazs, A. C.; Matyjaszewski, K., Stackable, Covalently Fused Gels: Repair and Composite Formation. Macromolecules 2015, 48 (4), 1169-1178.

25. Chen, S.; Phan-Thien, N.; Khoo, B. C.; Fan, X. J., Flow around Spheres by Dissipative Particle Dynamics. Phys. Fluids 2006, 18 (10), 103605.

26. Fan, H.; Striolo, A., Nanoparticle Effects on the Water-Oil Interfacial Tension. Phys. Rev. E 2012, 86 (5), 051610.

27. Liu, Y.; Yong, X.; McFarlin, G. t.; Kuksenok, O.; Aizenberg, J.; Balazs, A. C., Designing 
a Gel-Fiber Composite to Extract Nanoparticles from Solution. Soft Matter 2015, 11 (44), 86928700.

28. Salib, I.; Yong, X.; Crabb, E. J.; Moellers, N. M.; McFarlin, G. T. t.; Kuksenok, O.;

Balazs, A. C., Harnessing Fluid-Driven Vesicles to Pick up and Drop off Janus Particles. ACS Nano 2013, 7 (2), 1224-1238.

29. Lee, M. T.; Vishnyakov, A.; Neimark, A. V., Calculations of Critical Micelle Concentration by Dissipative Particle Dynamics Simulations: the Role of Chain Rigidity. J. Phys. Chem. B 2013, 117 (35), 10304-10310.

30. Fuchslin, R. M.; Fellermann, H.; Eriksson, A.; Ziock, H. J., Coarse Graining and Scaling in Dissipative Particle Dynamics. J. Chem. Phys. 2009, 130 (21), 214102.

31. Spaeth, J. R.; Kevrekidis, I. G.; Panagiotopoulos, A. Z., A Comparison of Implicit- and Explicit-Solvent Simulations of Self-Assembly in Block Copolymer and Solute Systems. $J$. Chem. Phys. 2011, 134 (16), 164902.

32. Nikoobakht, B.; El-Sayed, M. A., Preparation and Growth Mechanism of Gold Nanorods (NRs) Using Seed-Mediated Growth Method. Chem. Mater. 2003, 15 (10), 1957-1962.

33. Sega, M.; Sbragaglia, M.; Biferale, L.; Succi, S., Regularization of the Slip Length Divergence in Water Nanoflows by Inhomogeneities at the Angstrom Scale. Soft Matter 2013, 9 (35), 8526-8531.

34. Moseler, M.; Landman, U., Formation, Stability, and Breakup of Nanojets. Science 2000, 289 (5482), 1165-1170.

35. The Peclet number for the nanoparticle can be estimated as $P e=L V / D$, where $L$ is the diameter of the particle, $V$ is the particle velocity, and $D \approx 2.5 \times 10^{-4}$ is diffusion coefficient of 
the particle. For the strong shear $\dot{\gamma} \approx 1.67 \times 10^{-2}, V \approx 0.01$, which leads to a Peclet number of $P e \approx 400$. For the weakest shear $\dot{\gamma} \approx 1.67 \times 10^{-4}, V \approx 2 \times 10^{-4}$ and $P e \approx 8$.

36. With the shear rate of $8.33 \times 10^{-3}$ used in (c-d), we observe the release of the particle in all eight simulation runs. With the lower shear rate of $1.67 \times 10^{-3}$, we observe particle release in five out of eight runs (results not shown). 